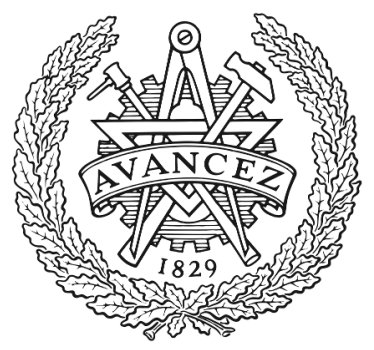

CHALMERS

UNIVERSITY OF TECHNOLOGY

\title{
Estimate of pre-thermal quench non-thermal electron density profile during Ar pellet shutdowns of low-density target plasmas in DIII-D
}

Downloaded from: https://research.chalmers.se, 2023-04-26 13:54 UTC

Citation for the original published paper (version of record):

Hollmann, E., Austin, M., Bykov, I. et al (2021). Estimate of pre-thermal quench non-thermal electron density profile during Ar pellet shutdowns of low-density target plasmas in DIII-D. Physics of Plasmas, 28(7). http://dx.doi.org/10.1063/5.0050903

N.B. When citing this work, cite the original published paper. 


\section{Estimate of pre-thermal quench non-thermal electron density profile during Ar pellet shutdowns of low-density target plasmas in DIII-D}

Cite as: Phys. Plasmas 28, 072501 (2021); https://doi.org/10.1063/5.0050903

Submitted: 24 March 2021. Accepted: 07 June 2021 . Published Online: 06 July 2021

E. M. Hollmann, (D) M. Austin, I. Bykov, N. W. Eidietis, (D) O. Embreus, J. L. Herfindal, (D) M. Hoppe, (D) A. Lvovskiy, (D) P. B. Parks, (D) C. Paz-Soldan, D. Shiraki, and I. Svenningsson
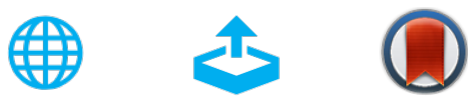

View Online

Export Citation

CrossMark

\section{ARTICLES YOU MAY BE INTERESTED IN}

Gyrokinetic investigation of the nonlinear interaction of Alfvén instabilities and energetic particle-driven geodesic acoustic modes

Physics of Plasmas 28, 072504 (2021); https://doi.org/10.1063/5.0049588

Radial runaway losses in tokamak disruptions

Physics of Plasmas 28, 032505 (2021); https://doi.org/10.1063/5.0032283

Equations and improved coefficients for parallel transport in multicomponent collisional plasmas: Method and application for tokamak modeling

Physics of Plasmas 28, 062308 (2021); https://doi.org/10.1063/5.0047618 


\title{
Estimate of pre-thermal quench non-thermal electron density profile during Ar pellet shutdowns of low-density target plasmas in DIII-D
}

Cite as: Phys. Plasmas 28, 072501 (2021); doi: 10.1063/5.0050903

Submitted: 24 March 2021 - Accepted: 7 June 2021 .

Published Online: 6 July 2021

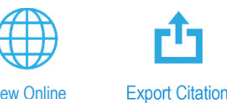

E. M. Hollmann, ${ }^{1, a)}$ (D M. Austin, ${ }^{2}$ (D) I. Bykov, ${ }^{3}$ N. W. Eidietis, ${ }^{3}$ O. Embreus, ${ }^{4}$ (D) J. L. Herfindal, ${ }^{5}$ M. Hoppe, ${ }^{4}$ A. Lvovskiy, ${ }^{3}$ (D) P. B. Parks, ${ }^{3}$ (iD C. Paz-Soldan, ${ }^{3}$ (iD D. Shiraki, ${ }^{5}$ and I. Svenningsson ${ }^{4}$

\author{
AFFILIATIONS \\ 'University of California San Diego, 9500 Gilman Drive, La Jolla, California 92093-0417, USA \\ ${ }^{2}$ University of Texas at Austin, P.O. Box 8058, Austin, Texas 78713, USA \\ ${ }^{3}$ Ceneral Atomics, P.O. Box 85608, San Diego, California 92186-5608, USA \\ ${ }^{4}$ Chalmers University of Technology, Gothenburg 412 96, Sweden \\ ${ }^{5}$ Oak Ridge National Laboratory, P.O. Box 2008, Oak Ridge, Tennessee 37831, USA \\ ${ }^{a}$ Author to whom correspondence should be addressed: ehollmann@ucsd.edu
}

\begin{abstract}
The radial density profile of pre-thermal quench (pre-TQ) early-time non-thermal (hot) electrons is estimated by combining electron cyclotron emission and soft $\mathrm{x}$-ray data during the rapid shutdown of low-density $\left(n_{e} \lesssim 10^{19} \mathrm{~m}^{-3}\right)$ DIII-D target plasmas with cryogenic argon pellet injection. This technique is mostly limited in these experiments to the pre-TQ phase and quickly loses validity during the TQ. Two different cases are studied: a high $(10 \mathrm{keV})$ temperature target and a low $(4 \mathrm{keV})$ temperature target. The results indicate that early-time, lowenergy $(\sim 10 \mathrm{keV})$ hot electrons form ahead of the argon pellet as it enters the plasma, affecting the pellet ablation rate; it is hypothesized that this may be caused by rapid cross field transport of argon ions ahead of the pellet or by rapid cross field transport of hot electrons. Fokker-Planck modeling of the two shots suggests that the hot electron current is quite significant during the pre-TQ phase (up to 50\% of the total current). Comparison between modeled pre-TQ hot electron current and post-TQ hot electron current inferred from avalanche theory suggests that hot electron current increases during the high-temperature target TQ but decreases during the low-temperature target TQ. The uncertainties in this estimate are large; however, if true, this suggests that TQ radial loss of hot electron current could be larger than previously estimated in DIII-D.
\end{abstract}

Published under an exclusive license by AIP Publishing. https://doi.org/10.1063/5.0050903

\section{INTRODUCTION}

Runaway electron (RE) current formation during tokamak disruptions is a critical area of research for magnetic fusion energy, as large post-disruption RE currents have the potential to cause unacceptably expensive wall damage in future large tokamak-based fusion reactors. ${ }^{1,2}$ However, designing methods to avoid or minimize potential RE wall damage in future large tokamaks like ITER is extremely challenging, as RE wall damage is a complex multi-step process.

Disruptions are global instabilities, which typically occur in tokamaks only during a rare loss of position control, during massive impurity injection, or when the discharge is pushed beyond stability boundaries in current, density, or pressure. Disruptions typically start with a pre-thermal quench (pre-TQ) phase characterized by edge cooling due to increasing impurity radiation, as well as by current profile contraction due to this edge cooling. Next, the thermal quench (TQ) occurs, during which global magnetohydrodynamic (MHD) modes cause rapid global energy loss to the wall, and core temperature collapse. Next, the current quench (CQ) occurs, during which the plasma toroidal thermal current decays away due to the higher cold plasma thermal resistivity. Finally, if sufficient non-thermal electrons are present in the plasma, a RE plateau will form, which is a long-lived current channel with current carried dominantly by REs. This regime has also been referred to as the "runaway beam regime."3

During the initial pre-thermal quench (pre-TQ) phase of a disruption, at least in "mitigated" disruptions with higher-Z impurity injection, edge plasma cooling due to impurity radiation creates hot 
(superthermal) seeds, dominantly by the "hot tail" mechanism, where rapid temperature collapse abandons hot electrons on the tail of the distribution. $^{6}$ Additionally, during the TQ phase, the large global MHD modes lead to the creation of stochastic field regions throughout the plasma, greatly increasing radial thermal conductivity and causing loss of hot electrons to the wall. ${ }^{7-9}$ In DIII-D, this TQ loss of hot electrons is thought to be fairly small (of order $10 \%-30 \%$ ), although this is based only on modeling or extremely indirect measurements. ${ }^{10,11}$ Post-TQ hot electron current is clearly seen to increase with increasing central plasma temperature, supporting the picture that the TQ is a net creator of superthermal electrons, i.e., more hot electrons are created by the hot tail mechanism than lost by radial transport during the TQ in mid-sized tokamaks. ${ }^{12}$ After the TQ, the resulting cold $\left(T_{e}\right.$ $\approx 5 \mathrm{eV}$ ) current quench (CQ) plasma is thought to heal stochastic regions rapidly (on a $0.1 \mathrm{~ms}$ timescale), resulting in greatly reduced radial loss of hot electrons during the CQ, although this belief is based entirely on simulations. ${ }^{8,9}$ There is experimental evidence that MHD modes or turbulence can cause loss of REs during the CQ, ${ }^{13}$ but this loss term has not been quantified and is typically ignored. The cold resistive CQ plasma and decaying toroidal current result in a large toroidal electric field, causing avalanche amplification of REs, so the CQ is normally thought of as being a RE amplification phase, with minimal radial loss, and with a (theoretically) well-understood RE gain given by knock-on avalanche. ${ }^{14}$ The formation of new superthermal electrons due to hot tail ${ }^{6}$ or Dreicer ${ }^{15}$ processes is normally ignored in modeling of DIII-D during the CQ. Following the CQ, the disruption can move into the RE plateau phase, where toroidal current is dominated by the REs, and then the RE final loss phase, where the $\mathrm{RE}$ beam strikes the wall and can cause localized wall damage. ${ }^{16}$ Despite this complex sequence of events, RE wall damage is thought to be ultimately proportional to the amount of REs surviving the TQ MHD, so pre-TQ and TQ RE formations and loss are extremely important components of this process.

The work presented here focuses on detailed data analysis and modeling of superthermal electron formation during the pre-TQ phase. Two different cases are studied: an "early" shutdown with a high initial central $T_{e} \approx 10 \mathrm{keV}$ and a "late" shutdown with a lower initial central $T_{e} \approx 4 \mathrm{keV}$. Both target plasmas are shutdown with a rapid $(V \sim 200 \mathrm{~m} / \mathrm{s})$ small ( 14 Torr $\mathrm{L} \approx 4 \times 10^{20}$ atoms) Ar pellet injection, resulting in very rapid $(\sim 1 \mathrm{~ms}$ timescale) radiative cooling and, eventually, RE plateau formation. The early shutdown contains significant non-thermal electrons prior to pellet injection, while the late shutdown does not. The origin of the non-thermal electrons in the early shutdown case is not studied here but is assumed to arise by slide-away during discharge start-up. ${ }^{3}$ For simplicity, no distinction will be made here between slide-away, superthermal seed, and runaway electrons: ${ }^{3}$ the thermal (Maxwellian) higher density bulk electrons (starting at $4-10 \mathrm{keV}$ and dropping down to $5 \mathrm{eV}$ during the shutdown) will be referred to as "cold" electrons, while the lower density, hotter superthermal tail (starting at around $4-10 \mathrm{keV}$ and moving up in energy during the shutdown) will be referred to as "hot" electrons.

The main new results presented in this work can be summarized as follows:

(a) Pre-TQ radial profiles of hot electrons formed by the Ar pellet injection are reconstructed for the first time by combining electron cyclotron emission (ECE) and soft x-ray (SXR) profiles. Large pre-TQ hot electron density fractions of up to 0.1 and hot electron temperatures up to about $10 \mathrm{keV}$ are reconstructed. The uncertainties in these fits are fairly large during the pre-TQ (of order 50\%) and grow even larger (past order unity) during the TQ.

(b) Hot electrons appear to exist ahead of the argon pellet, possibly due to radial transport of argon ions or hot electrons moving ahead of the pellet. These hot electrons appear to dominate the argon pellet ablation, i.e., thermal ablation modeling does not match the pellet light curves.

(c) Pre-existing hot electrons in the early shutdown target plasma appear to somewhat reduce the formation rate of additional hot electrons during the Ar pellet injection. According to the modeling, this occurs because the hot electrons are present in sufficient numbers to carry non-negligible plasma current, reducing the electric field resulting from thermal plasma cooling.

(d) Based on the modeling, the TQ in the early shutdown shot appears to increase hot electron current, while in the late shutdown shot, the TQ appears to cause a decrease of hot electron current. This suggests that the disruption TQ can serve to either increase or decrease hot electron current, depending on the TQ conditions and MHD. The late shutdown has higher $I_{P}$ and lower $q$, resulting in more MHD drive, possibly explaining this observation. The uncertainties in this observation are extremely large, as discussed in the text. If true, however, this indicates that TQ RE losses in DIII-D could be larger than the previously estimated $10 \%-30 \%$.

\section{EXPERIMENTAL SETUP}

Top and side views of the DIII-D tokamak highlighting key hardware components used here are shown in Figs. 1(a) and 1(b). ${ }^{17}$ These experiments were performed with inner wall-limited (IWL) deuterium-fueled target plasmas. Early internal transport barrier (ITB) plasma shots are used, where low electron densities $\left(n_{e} \lesssim 10^{19} \mathrm{~m}^{-3}\right)$ and high early gyrotron heating during Ohmic ramp-up allow transient achievement of extremely high (up to $10 \mathrm{keV})$ central $(\rho<0.5)$ electron temperature early in the discharge. The "early" shutdowns occur while the ITB exists, while the "late" shutdowns occur after the

\section{(a) Top view}

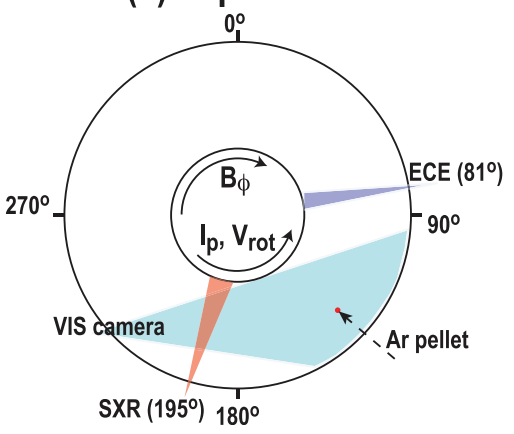

(b) Side view

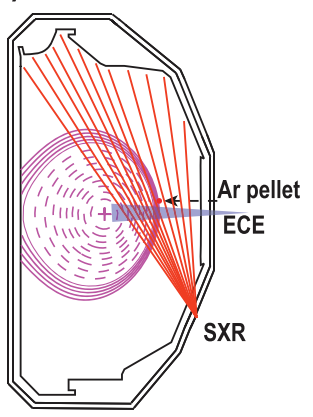

FIG. 1. Schematic of DIII-D tokamak from (a) top view and (b) side view, showing some essential diagnostics and Ar pellet injection. 
ITB has ended. Additional details of these discharges were provided in a previous study. ${ }^{12}$

The main diagnostics used in this work are a fast-framing visible camera, electron cyclotron emission (ECE), and a soft x-ray (SXR) array. The visible camera is bandpass filtered to isolate Ar-I $695 \mathrm{~nm}$ emission with a $5 \mathrm{~nm}$ bandpass and is aimed to view the injected $\mathrm{Ar}$ pellet injection region to measure neutral argon ablation and estimate local argon deposition into the plasma. Absolute calibration of the camera data is obtained using a calibrated light source. The ECE diagnostic measures $2^{\text {nd }}$ harmonic electron cyclotron emission from the plasma outer midplane with 40 frequency bands. ${ }^{18}$ The SXR diagnostic measures plasma bremsstrahlung emission in the $2-10 \mathrm{keV}$ energy range with 12 view chords. ${ }^{19}$

Figure 2 shows time traces giving an overview of the two cases studied here. In both cases, argon pellet injection is used to terminate the discharge, causing strong hot electron formation and RE plateau formation, as shown by the plasma current traces in Fig. 2(a) and hard $\mathrm{x}$-ray (HXR) emission traces in Fig. 2(b). In the "early shutdown" shot
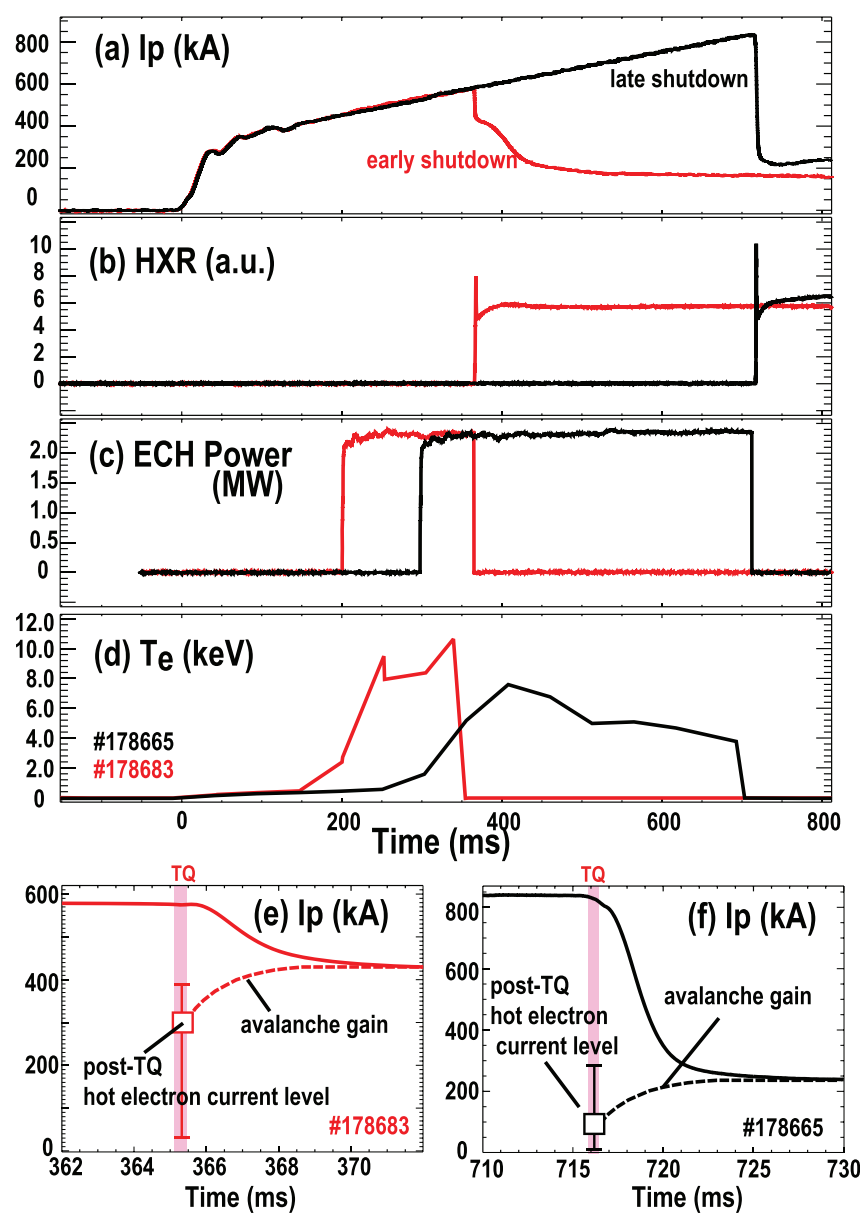

FIG. 2. Time traces from the two shots studied here showing overviews of (a) plasma current, (b) hard X-ray (HXR) emission, (c) ECH input power, and (d) central (thermal, cold) electron temperature. Zoomed time traces of plasma current are shown for the two shots in (e) and (f) showing estimated hot electron current.
No. 178683, pellet injection occurs at $\mathrm{t}=364 \mathrm{~ms}$ when mean (peak) cold electron temperature is $6 \mathrm{keV}(10 \mathrm{keV})$. In the "late shutdown" shot No. 178665, pellet injection occurs at $t=715 \mathrm{~ms}$ when mean (peak) cold electron temperature is $2 \mathrm{keV}(4 \mathrm{keV})$. Electron cyclotron heating $(\mathrm{ECH})$ power is turned on earlier in the early shutdown case; this is done to create more early hot non-thermal electrons at the time of the shutdown, relative to the late shutdown case.

The standard method for estimating post-TQ hot electron current in DIII-D disruptions is to ignore radial loss and new hot electron formation, in which case hot electron current evolution is, typically, dominated by avalanche gain. ${ }^{11,12}$ In this case, the post-TQ hot electron current can be estimated from RE plateau current at the end of the CQ and from the avalanche growth rate; ${ }^{14}$ in these two shots, this method gives post-TQ hot electron currents of about $300 \mathrm{kA}$ (early shutdown) and $100 \mathrm{kA}$ (late shutdown), as shown in Figs. 2(d) and 2(e). The increased avalanche rate due to partial screening is not included here; ${ }^{20}$ this correction is not thought to be large in these shots due to the low plasma current and low resulting loop voltage. ${ }^{12}$ As discussed later, it is possible that Dreicer hot electron formation is not negligible during the CQ of these shots; this results in significant uncertainty in the post-TQ hot electron current, as indicated by the estimated error bars in Figs. 2(d) and 2(e).

Figure 3 shows radial profiles of the two cases studied here as a function of normalized minor radius $r / a$. Temperature profiles shortly before pellet injection are shown in Figs. 3(a) and 3(b). It can be seen that the early shutdown case appears to have a hot electron population even before pellet injection at larger radii $r / a>0.5$. This can be seen by the larger apparent temperature seen in ECE when compared with Thomson scattering (TS) in Fig. 3(a). TS is expected to be dominantly sensitive to cold electrons, while ECE is more sensitive to hot electrons. The ECE data used here are not time-smoothed but represent single time steps from the $500 \mathrm{kHz}$ diagnostic. Error bars on the TS data are estimated from the quality of the fits to the spectra from the polychromators, while characteristic error bars on the ECE data are estimated from the level of fluctuations over time over a $10 \mathrm{~ms}$ window.

Figure 3(c) compares the total argon deposition profile to the thermal (TS) temperature profiles for early and late shutdown. This comparison indicates that the early shutdown causes more rapid pellet ablation in the edge, despite having lower thermal $T_{e}$, suggesting that the hot electrons could be dominating Ar pellet ablation. As will be discussed later, this is supported by Ar pellet ablation rate analysis, i.e., the pellet ablation does appear to be heavily influenced by hot electrons. The argon deposition profiles in Fig. 3(c) are obtained from the lineintegrated Ar-I brightness from the camera images but assuming that the photon efficiency (ionizations/photon, SXB) is a constant vs time, so that total spatially integrated Ar-I brightness is proportional to the ionization source term. The normalization is then obtained from the known initial argon pellet volume. The use of a single constant $S / X B$ value is reasonably well supported by previous analysis using absolutely calibrated Ar-I imaging of argon pellet ablation in DIII-D and atomic physics modeling, finding that the Ar-I emission comes from plasma near the pellet with electron density $n_{e} \approx 10^{21} / \mathrm{m}^{3}$ and electron temperature $T_{e} \approx 0.4-0.6 \mathrm{eV}$, giving $\mathrm{S} / \mathrm{XB} \approx 70-150$, i.e., about $2 \times$ uncertainty. ${ }^{11}$ Use of a constant $\mathrm{S} / \mathrm{XB}$ is also qualitatively supported by cryogenic pellet ablation simulations, which indicate that the neutral/ion transition boundary tends to be pinned at a low electron temperature regardless of upstream temperature. ${ }^{21}$ 

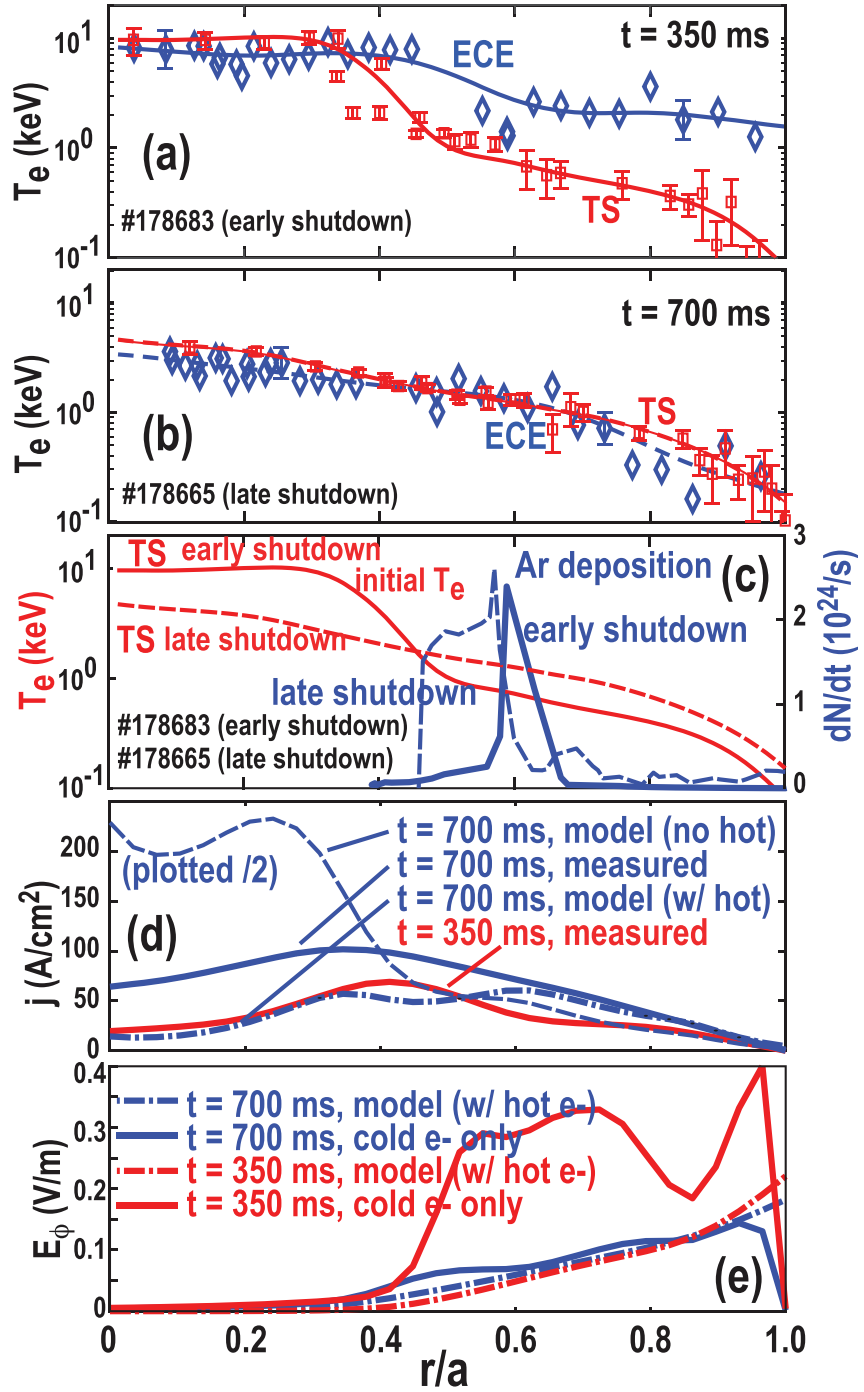

FIG. 3. Radial profiles of electron temperature from ECE and Thomson scattering (TS) taken $\sim 15 \mathrm{~ms}$ before argon pellet shutdown for (a) early shutdown and (b) late shutdown; (c) comparison of argon deposition profiles and TS temperature profiles; (d) current density profiles measured with the motional Stark effect (MSE) and predicted with diffusive model (including and not including hot electrons) at $t=700 \mathrm{~ms}$ for late case and at $t=350 \mathrm{~ms}$ for early case; and (e) toroidal electric field profiles based on thermal electron resistivity only and from diffusive model including hot electrons at $t=700 \mathrm{~ms}$ for late case and at $t=350 \mathrm{~ms}$ for early case.

Figure 3(d) shows measured current density profiles (solid curves). These are measured during shots, which had neutral beam blips at times just before shutdown, allowing motional Stark effect (MSE) constrained equilibrium reconstructions of the current density profile. The ECE/TS discrepancy seen in Fig. 3(a) suggests that some of the plasma current could be carried by hot electrons in these shots; the ECE/TS discrepancy lasts up to the shutdown at $365 \mathrm{~ms}$ in the early shutdown case and up to about $500 \mathrm{~ms}$ in the late shutdown case, well before the shutdown around $715 \mathrm{~ms}$. The measured current density profiles, when compared with a 1D electric field diffusion model, support the presence of non-negligible hot current at early times $(<500 \mathrm{~ms})$. The electric field diffusion model uses the measured electric field at the wall as a boundary condition and then diffuses the electric field inward from the beginning of the shot, using the TS and EFIT profiles to estimate plasma thermal resistivity. Assuming no hot electrons gives centrally peaked current density profiles with central magnitude about $8 \times$ larger than measured at $t=700 \mathrm{~ms}$ in the late shutdown case, as shown by the dashed line in Fig. 3(d). Using a rough model for hot electrons gives a current density profile much closer to the data, as shown by the dotted-dashed curve in Fig. 3(d). As a rough model for hot electrons, the hot electron temperature is assumed to be the cold electron temperature times a linear scale factor going from 0 on center to 5 at $r / a=1$ (so, e.g., a thermal temperature of $1 \mathrm{keV}$ gives a hot temperature of $5 \mathrm{keV}$ at the edge). This radially increasing scale factor is motivated by ECE + SXR reconstructions (discussed later), which indicate very little hot electrons on center initially, but significant hot electrons toward the outer edge of the plasma, with temperature perhaps $5-10 \times$ the cold electron temperature. The hot electrons are removed at time $t=500 \mathrm{~ms}$. The conductivities of hot and cold components are simply added together to approximate total conductivity. Conductivity is estimated including the neo-classical reduction for trapped particles. ${ }^{22-24}$ Purely Ohmic conductivity is assumed, i.e., $V \times B$ and $J \times B$ terms in the generalized Ohm's Law are expected to be small here and are ignored. It is not attempted here to fine-tune the hot electron model to perfectly match the data, although a sensitivity scan was done to verify that the need for hot electrons to match central current is robust.

Figure 3(e) shows the estimated toroidal electric field vs radius for the late case at $t=700 \mathrm{~ms}$ and for the early case at $t=350 \mathrm{~ms}$, i.e., about $15 \mathrm{~ms}$ before the pellet impact in both cases. The electric field profile is crucial for simulating hot electron formation. The dotteddashed curve shows the electric field profile from the diffusive model including hot electrons. The solid curves show the electric field from Ohm's Law, i.e., the measured current profile times the calculated thermal electron resistivities. This is typically considered a good estimate for $E_{\phi}$ but will be inaccurate in the presence of non-thermals. Consistent with this, the early case shows a much higher Ohm's Law $E_{\phi}$ than the diffusive model; comparing the curves suggests that at early times, the effective resistivity is actually about two times lower than the thermal resistivity, owing to the presence of hot electrons.

\section{RECONSTRUCTION OF PRE-TQ RE PROFILES FROM ECE + SXR DATA}

Pre-TQ profiles of non-thermal (RE) electrons are reconstructed by using ECE and SXR data. The analysis sequence is:

(A) The electron velocity distribution is approximated with two temperatures, i.e., the electrons are separated into a hot component $\left(n_{e, \text { hot }}, T_{e, \text { hot }}\right)$ and a cold component $\left(n_{e, \text { cold }}, T_{e, \text { cold }}\right)$ at each radius.

(B) The cold electron temperature $T_{e, \text { cold }}$ is taken from an exponential decay fit to ECE data during the shutdown, scaled to initial TS data, as described below. The exponential fit separates out cold and hot ECE temperature components $T_{e, \text { cold }}(\mathrm{ECE})$ and $T_{e, h o t}(\mathrm{ECE})$. It is assumed that normal optically thick ECE analysis is still valid for the cold component, $T_{e, \text { cold }}(\mathrm{ECE})=T_{e, \text { cold }}$. However, for the hot ECE temperature, 
$T_{e, h o t}(\mathrm{ECE})<T_{e, h o t}$, since the hot component is optically gray and also due to re-absorption by the cold component.

(C) The cold electron density $n_{e, \text { cold }}$ is taken from the initial TS $n_{e}$ profile, plus the electrons estimated to be added from the Ar pellet from fast camera data, poloidally averaged. Three different limiting cases of Ar transport are considered and discussed in the text.

(D) $n_{e, h o t}$ and $T_{e, h o t}$ remain as unknowns. The hot ECE component depends on $\left(n_{e, h o t}, T_{e, h o t}\right)$ and $\left(n_{e, \text { cold }}, T_{e, \text { cold }}\right)$. The total SXR signal also depends on $\left(n_{e, h o t}, T_{e, h o t}\right)$ and $\left(n_{e, \text { cold }}, T_{e, \text { cold }}\right)$. In this case, the total signal is a simple addition of the two bremsstrahlung components. However, the signal is line-integrated, so the variables cannot be solved locally. $\left(n_{e, h o t}, T_{e, h o t}\right)$ profiles are varied at each time step to try to produce best matches to the measured ECE and SXR profiles.

To justify step (A) above, there is presently no diagnostic able to measure the electron distribution function accurately during disruptions. However, the use of a two-temperature fit is reasonably accurate during the pre-TQ phase according to simulations. Figure 4 shows electron distribution functions as a function of (a) parallel momentum and (b) kinetic energy at four different time steps simulated by CODE Fokker-Planck simulations. ${ }^{25,26}$ Two-temperature fits are shown in Fig. 4(b), demonstrating that the distribution functions can be well described using two-temperature fits during the earlier stages of the disruption (before the CQ and RE plateau formations). For the simulations of Fig. 4, relatively typical parameters from these experiments are

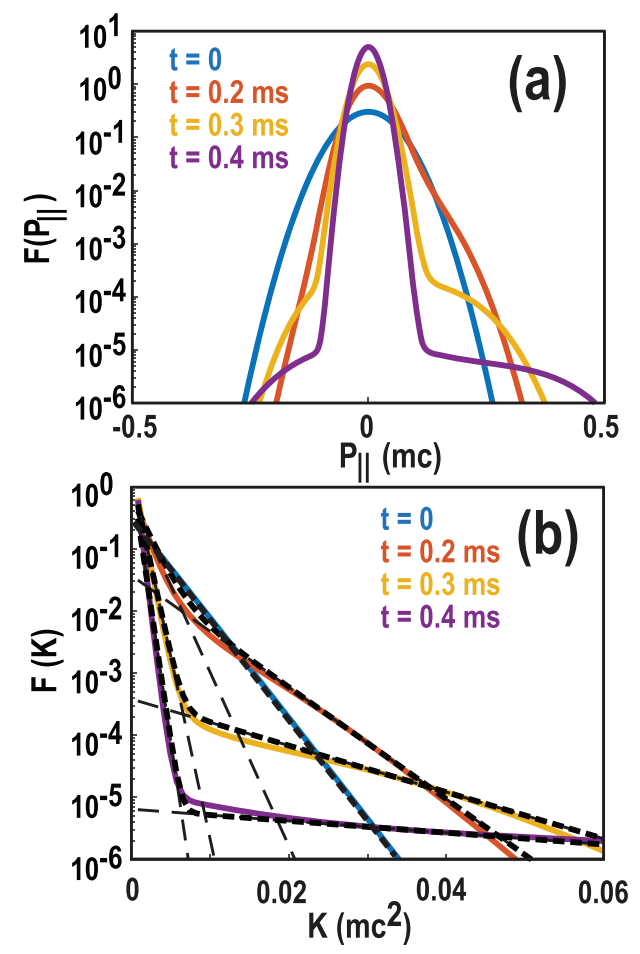

FIG. 4. (a) Sample electron distribution function as different time steps simulated by CODE as a function of normalized parallel momentum and (b) the same distribution function as a function of normalized kinetic energy and two-temperature fits. used: initial electric field $=0.05 \mathrm{~V} / \mathrm{m}$, initial electron temperature $=1.4 \mathrm{keV}$, initial electron density $=4 \times 10^{18} \mathrm{~m}^{-3}$, and temperature collapse timescale of $0.2 \mathrm{~ms}$.

For step (B) above, at each radius, the initial (pre-shutdown) non-thermal (hot) ECE signal level can be estimated by comparing TS with ECE profiles, as done in Figs. 3(a) and 3(b). The subsequent thermal ECE signal evolution is estimated by assuming that the cold (thermal) ECE signal drops exponentially from its initial value to $T_{e}=5 \mathrm{eV}$ by the start of the CQ; this is based on TS measurements of the CQ temperature, typically giving $T_{e} \approx 5 \mathrm{eV}$. An exponential decay is used, as this is reasonably close to the available TS data of the temperature collapse shape during disruptions. This low $(5 \mathrm{eV})$ value for the CQ temperature in DIII-D has also been independently supported with a variety of cross-checks, including wall probes, spectroscopy, and CQ current decay rate. $^{27}$ The precise CQ temperature (e.g., changing from 5 to 3 or to $8 \mathrm{eV})$ causes negligible $(\sim 1 \%)$ change in the resulting reconstructed pre-TQ hot electron profiles, which are the main focus of this paper. However, the precise CQ temperature does have a significant effect on the predicted Dreicer formation rate during the CQ, discussed later, changing the rate by $2 \times$ with only several $\mathrm{eV}$ change in CQ temperature.

A fundamental assumption of this technique is that ECE and SXR signals are more strongly increased by hot electrons than TS signals. This is expected to be true for typical parameters of these experiments, as illustrated in Fig. 5, which shows predicted ECE, SXR, and TS signals for the simulated distribution functions of Fig. 4. Figures 5(a) and 5(b) show the result of the two-temperature fits at 4 time steps, showing (a) cold and hot electron densities and (b) cold and hot electron temperatures. Figure 5(c) shows predicted ECE brightness temperature for cold plasma only (blue) and for (red) the total distribution including hot electrons. A similar plot is shown in Fig. 5(d) for SXR emissivity. Figure 5(e) then shows predicted TS temperature fits. It can be seen that ECE and SXR are typically increased significantly (about $50 \%$ ) by the presence of expected levels of hot electrons. ECE brightness temperature will trend toward the hot signal, depending on the hot electron density, resulting in a large perturbation. SXR has a roughly $2 \mathrm{keV}$ high-pass filter and is, therefore, very sensitive to hot electrons during the pre-TQ, when the cold temperature drops below $2 \mathrm{keV}$ and the hot temperature rises above $2 \mathrm{keV}$, as shown in Fig. 5(b). TS, on the other hand, is increased by at most $10 \%$, more typically less than $1 \%$. This is because TS wavelength bins are optimized to measure temperatures of about $5 \mathrm{keV}$ or less. Also, the least square fits to the counts in each wavelength bin to arrive at a temperature will tend to weight the cold temperature much more heavily, due to the significantly higher cold density. TS wavelength bins and sample counts for two different cold temperatures are shown in Fig. 5(f).

Examples of the exponential fits to ECE data are shown in Fig. 6. The start of the exponential decay is taken as the time at which $T_{e}$ begins to deviate strongly (up or down) from its initial steady (on a ms timescale) value, shown by dashed lines in Fig. 6. Figure 6(a) shows a low minor radius time trace where initial non-thermal signal is small. The time trace is seen to begin dropping suddenly, at which time, an exponential fit is applied (blue curve), giving an estimated cold component vs time. Figure $6(\mathrm{~b})$ shows a large minor radius time trace where the initial non-thermal signal is large. The time trace is seen to begin rising suddenly, at which time, an exponential decay is also applied to estimate the time behavior of the cold component. 

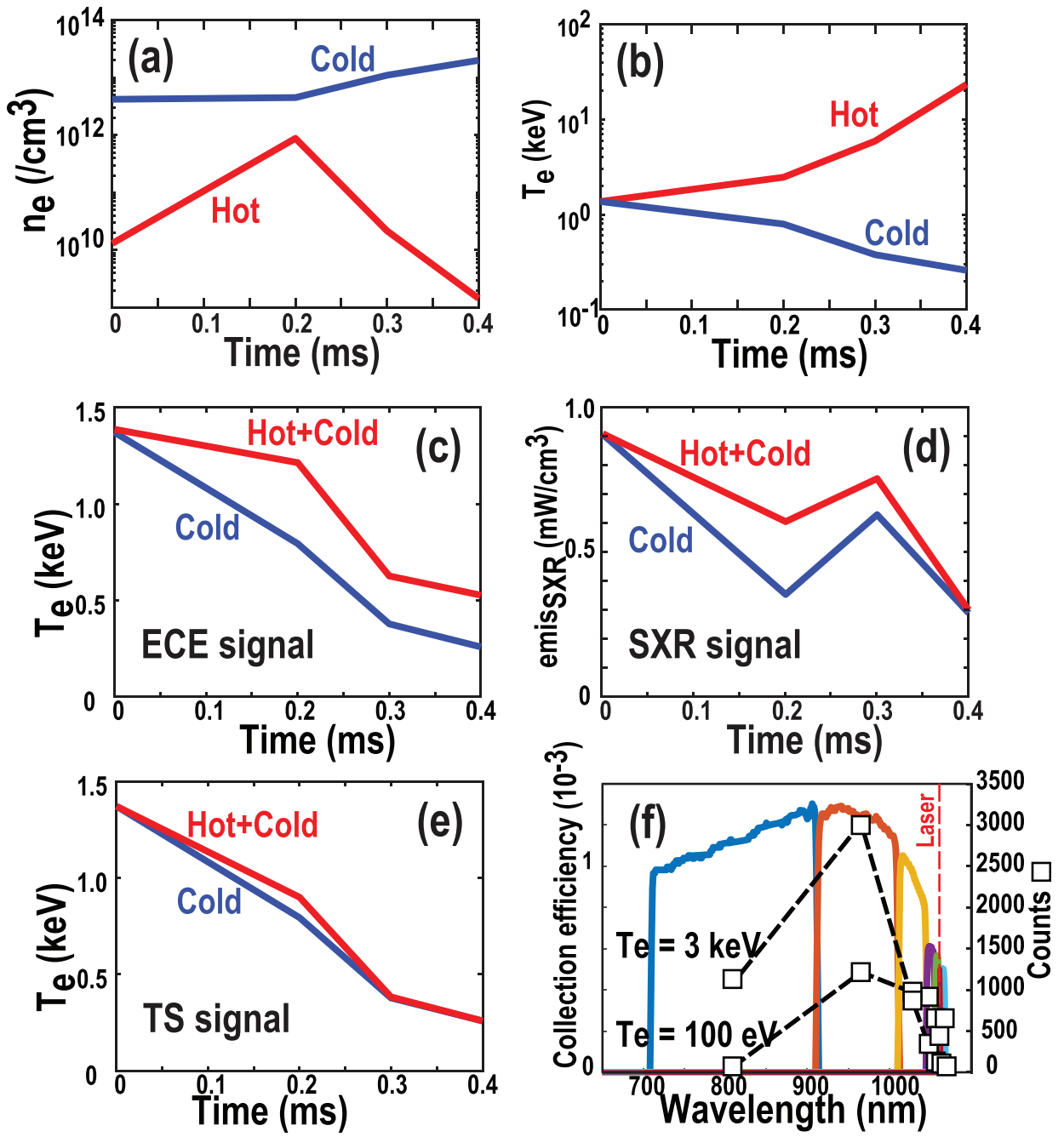

FIG. 5. (a) Hot and cold electron density and (b) hot and cold electron temperature vs time from the two-temperature fits to simulated distribution function of Fig. 4, as well as predicted resulting diagnostic signals from (c) ECE, (d) SXR, and (e) TS. Wavelength bins (color curves) and sample counts (dashed lines for two cold temperatures) are shown in (f).

The ECE exponential decay fit method to estimate $T_{e, \text { cold }}$ can be cross-checked at some isolated time slices with TS data, indicating it is reasonably valid. Figure 7 gives an example of (red) initial TS and ECE (cold component) radial temperature profiles as well as (black) midTQ TS and ECE (cold component) radial temperature profiles, showing good agreement within the scatter of the TS data.

For step (C) above, the initial cold electron density $n_{e, \text { cold }}$ is taken from the pre-shutdown TS profile. This assumes $n_{e, \text { hot }} \ll n_{e, \text { cold }}$ before the Ar pellet impact, since TS is not expected to be very sensitive to the hot $(10 \mathrm{keV})$ electrons, as discussed above. As described above, the Ar pellet ablation rate can be estimated from absolutely calibrated Ar-I line emission imaging. The increase in $n_{e, \text { cold }}$ with time is estimated using the additional electrons from this deposited argon, assuming instantaneous Ar charge-state equilibration with the local $T_{e, \text { cold. }}$ Ar ion transport (and associated additional cold electron transport, assuming ambipolarity) is investigated in three limits, as will be discussed below.

For step (D) above, the ECE and SXR data are used. The hot ECE temperature obtained with the two-temperature fit described above is not the actual hot temperature. These shots have very low electron density $n_{e} \lesssim 10^{19} \mathrm{~m}^{-3}$, so ECE density cutoff is not observed in the two cases selected here and is neglected. However, since the lowdensity hot electrons are optically gray, the hot ECE signal will go up with both hot temperature and hot density and is, therefore, underconstrained. Additionally, re-absorption of the hot ECE signal will depend on cold electron density and temperature, further complicating analysis. To help constrain the solution, line-integrated SXR data are added into the analysis. Figure 8 shows radial profiles of (a) $T_{e}$ of the hot component and (b) $n_{e}$ of the hot component as well as resulting fits to (c) the hot ECE component (vs normalized radius) and (d) total line-integrated SXR brightness (as a function of view chord tangency radius). As shown in Fig. 8, fits to the data within about $2 \times$ are achieved by the optimization routine.

During the pre-TQ and TQ, injected Ar transport is quite complex and $3 \mathrm{D}$, with rapid $(\sim 1 \mathrm{~ms})$ toroidal expansion on helical field lines and somewhat slower ( several $\mathrm{ms})$ poloidal and radial transport of Ar ions. To make this complex situation tractable in the ECE + SXR fitting method, three different limiting cases of impurity 


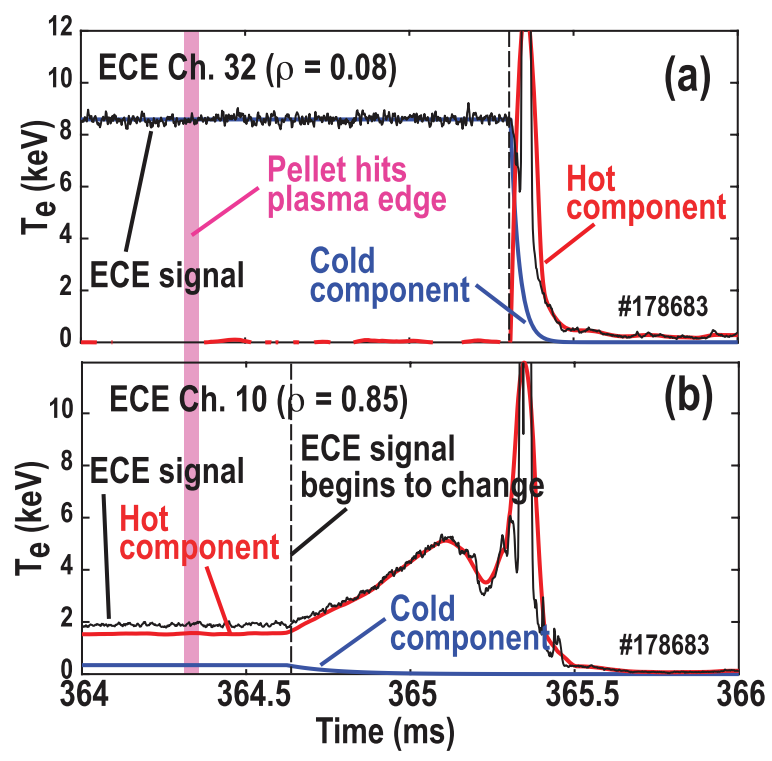

FIG. 6. Exponential fit method for analyzing ECE data with nonthermal spikes. Examples are shown at two different radii for "early" shutdown: (a) $\rho=0.08$ and (b) $\rho=0.85$. Exponential fit method for analyzing ECE data with nonthermal spikes. Examples are shown to diverge from its initially constant (on a $1 \mathrm{~ms}$ timescale) value. Black curves show ECE data, blue curves show cold component fit, and red curves show hot component (the total fit signal is the sum of the hot and cold components).

(Ar ion) transport are considered: no transport, instant toroidal transport, and instant toroidal transport + slow radial transport. In the "no transport" assumption, electron density at the ECE and SXR diagnostics just remains constant throughout the shutdown, i.e., it is assumed that argon ions never reach these diagnostics. In the "instant toroidal transport" assumption, it is assumed that ablated Ar ions instantly fill the flux surface occupied by the pellet. In the "diffusive assumption," instant toroidal transport is assumed as well as a reasonable radial $\mathrm{Ar}$ ion diffusion coefficient of $5 \mathrm{~m}^{2} / \mathrm{s}$. Dashed lines in Fig. 8 show the results of these different limiting approximations. Overall, it is found

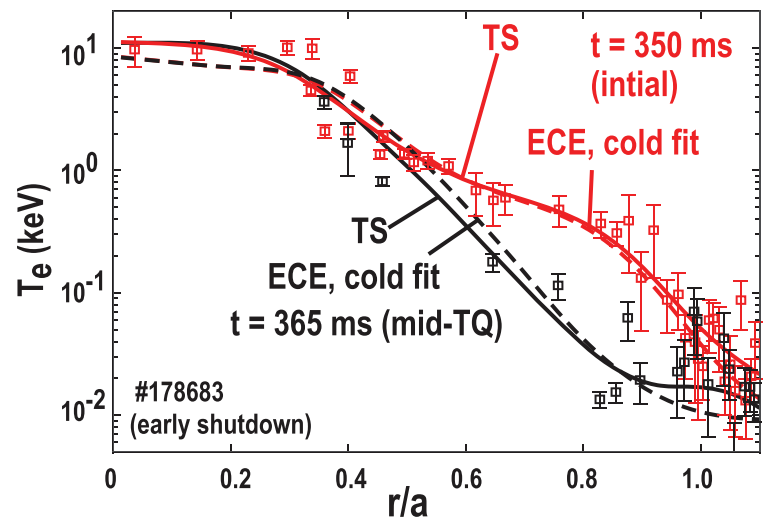

FIG. 7. Initial and mid-TQ radial electron temperature profiles from TS and ECE (cold component fit). Solid curves and squares are TS fit and data, respectively, while dashed curves are ECE cold component (without hot electron) fit.
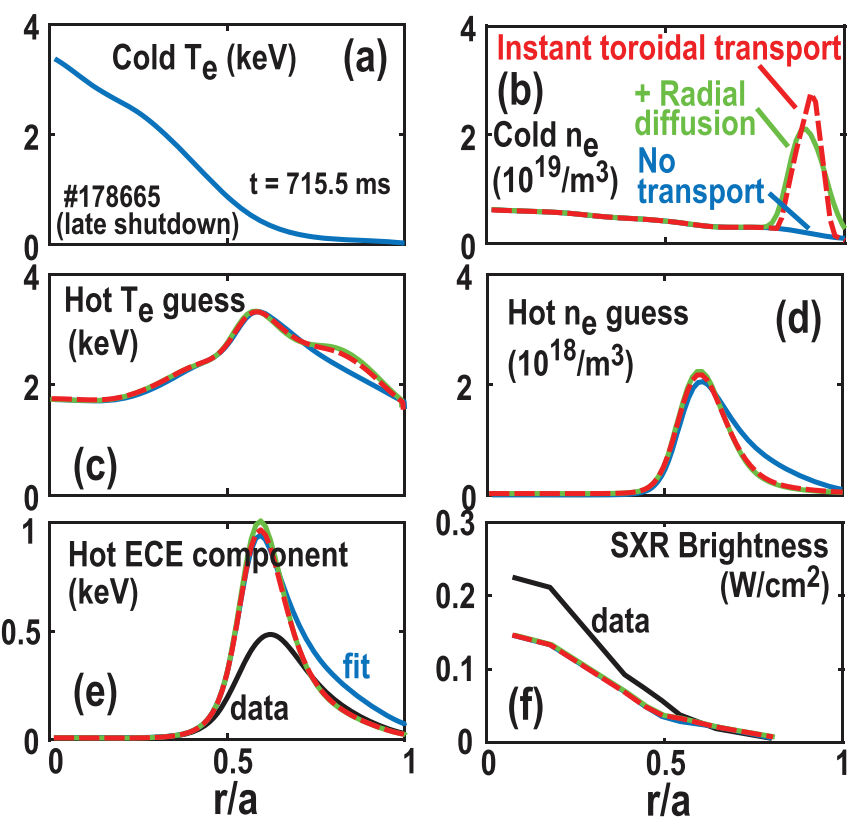

FIG. 8. Fits to hot temperature and density at one time step showing (a) cold temperature profile, (b) cold electron density profiles (for three different assumptions of Ar transport discussed in text), (c) resulting fits to hot electron temperature, (d) resulting fits to hot electron density, (e) hot ECE component data (black curve) and fits (colored curves), and (f) SXR brightness data and fits.

that the variations in Ar transport cause very little uncertainty in the reconstructed pre-TQ peak hot density and temperatures, but larger uncertainty at large radii (behind the pellet) and at later times (during the TQ). These uncertainties are propagated through the analysis and added as error bars in subsequent figures, adding to estimated uncertainty from ECE + SXR fit errors.

The total ECE signal resulting from a given electron plasma $\left(n_{e, \text { hot }}, T_{e, \text { hot }}\right)$ plus $\left(n_{e, \text { cold }}, T_{e, \text { cold }}\right)$ is estimated using the $2^{\text {nd }}$ harmonic $\mathrm{X}$-mode ECE absorption coefficient for weakly relativistic plasma. ${ }^{28}$ Absorption and emission from both hot and cold plasmas at a given frequency are included to estimate the total ECE radial profile; the predicted hot ECE temperature of Fig. 8(c) can then be obtained by subtracting the cold $T_{e}$ value. A $1 \mathrm{D}$ multiple bounce model is assumed with 11 bounces and a wall reflection coefficient of $R_{E C E}=0.76$. $^{29}$ Parametric scans indicate that the expected uncertainty in wall reflection coefficient $R_{E C E} \approx 0.7-0.8$ gives a fairly small (roughly linear) uncertainty in the predicted hot electron ECE signal from the model and is ignored here. Non-local emission is included in the ECE analysis, but harmonics outside of the 2nd harmonic are not included.

The SXR brightness profiles are modeled using the known diagnostic spectral sensitivity vs energy together with the theoretical bremsstrahlung spectral shape. ${ }^{30}$ To determine the effective charge state, $Z_{\text {eff }}$, the fully stripped carbon density measured from charge exchange recombination (CER) is used; the resulting carbon density is assumed constant. To best fit the initial SXR profiles, a small initial argon fraction $f_{A r}$ is also allowed; this initial argon fraction is assumed constant vs radius and time, and perturbs the SXR signals both by raising $Z_{\text {eff }}$ and through the $\mathrm{Ar} \mathrm{K} \alpha$ emission, which is calculated using the 
PrismSPECT. ${ }^{31} n_{e, \text { cold }}$ is estimated in the toroidally uniform limit as described above.

Reconstructed radial profiles of electron density and electron temperature for the early time shutdown at two time steps are shown in Fig. 9. The first time step, $t=364.4 \mathrm{~ms}$, is at the moment of pellet impact at the plasma edge, while the second plotted time step, $\mathrm{t}=365.1 \mathrm{~ms}$, is during the pre-TQ phase, when the pellet is at $\rho=0.65$. The location of the argon pellet at both time steps is shown with vertical green lines. The cold (and hot) electron temperatures reconstructed using the ECE + SXR fitting method are shown in Fig. 9(a) with black (red) curves. The hot electron density is shown in Fig. 9(b) with red curves, while the black curves show cold electron density. It can be seen that hot electrons with $T_{e} \approx 10 \mathrm{keV}$ already exist in the plasma when the argon pellet arrives. During the pre-TQ, as the argon pellet moves into the plasma edge, only a slight increase in non-thermal density occurs.

The blue curves in Fig. 9 show hot electron density profiles from CODE. Since CODE has no spatial dependence and no cross field transport, separate simulations were run at 9 radii. The initial hot temperature was taken to be $10 \mathrm{keV}$, and the initial hot density profile was taken from the data, the red-dashed line of Fig. 9(b). The slight difference between blue- and red-dashed lines in Fig. 9(b) is due to the definition of hot density used in CODE: an integral in velocity from the point of deviation from the initial cold distribution function is used, rather than an integral over the full hot Maxwellian (since CODE incorporates both Maxwellians into a single electron distribution function). For estimating the electric field, it is assumed the initial measured current profile does not evolve during the simulation, so the local electric field is estimated from the local current density times the local resistivity (to both hot and cold electrons). This is thought to be a reasonable approximation during the pre-TQ, where very little radial diffusion of current is expected. Consistent with the experiment, it can

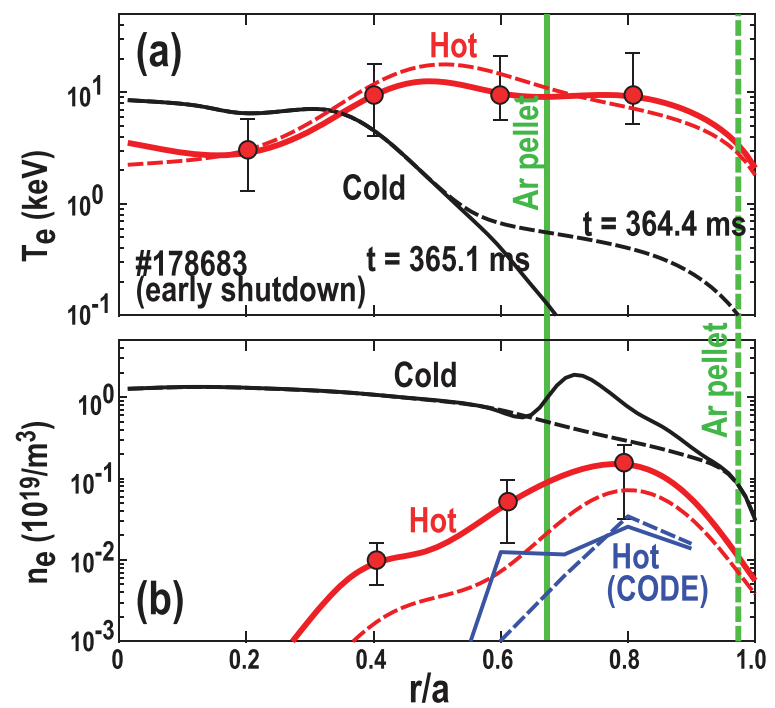

FIG. 9. Reconstructed radial profiles of (a) thermal and non-thermal electron temperature and $(b)$ thermal and non-thermal electron density at two times steps during the early shutdown shot (one at the pellet first impact and one during the pre-TQ). Blue curves show CODE simulations. be seen that the hot electron population experiences only a slight increase during the pre-TQ phase.

Figure 10 shows reconstructed radial profiles of electron density and electron temperature for the late time shutdown at two times (again at times corresponding to first argon pellet impact and during the pre-TQ). Unlike in early time shutdown, no hot electrons appear to be present when the argon pellet arrives. However, soon after the pellet arrival, strong formation of hot electrons with $T_{e} \approx 5 \mathrm{keV}$ occurs. Interestingly, the hot electrons appear to form well ahead of the pellet, indicating fast radial transport of either Ar ions or fast electrons ahead of the pellet. CODE simulations are again shown by blue curves. Similar to Fig. 9, the simulations are done independently at 9 radii and use the measured initial current profile to estimate the electric field profile during the pre-TQ. Unlike the data of Fig. 9, no initial hot seed is assumed, although hot electrons do appear in the simulation rapidly at early times, as shown by the blue-dashed curve in Fig. 10(b). It can be seen that the simulations find a reasonable match to the measured hot electron formation, being only about $2 \times$ lower than the data on peak.

The data and analysis of Figs. 9 and 10 are from two shots: $\# 178683$ as an early shutdown example and \#178665 as a late shutdown example. Similar ECE + SXR analysis performed on similar shots shows similar trends to those discussed above. Figure 11 gives an idea of the level of shot-shot scatter by showing results from 8 different shots (4 early shutdown and 4 late shutdown shots) with nominally repeated conditions (fueling, heating, current ramp, etc), arranged in order of increasing post-TQ RE current, Fig. 11(a). It can be seen that the early shutdown case appears to generate $2-3 \times$ more post-TQ hot current than the late shutdown case. Figure 11(b) shows the peak measured $n=1$ magnetic fluctuation level at the wall, showing no clear trends. Figure 11(c) shows the profile-averaged hot electron density immediately before the TQ from the ECE + SXR analysis, showing

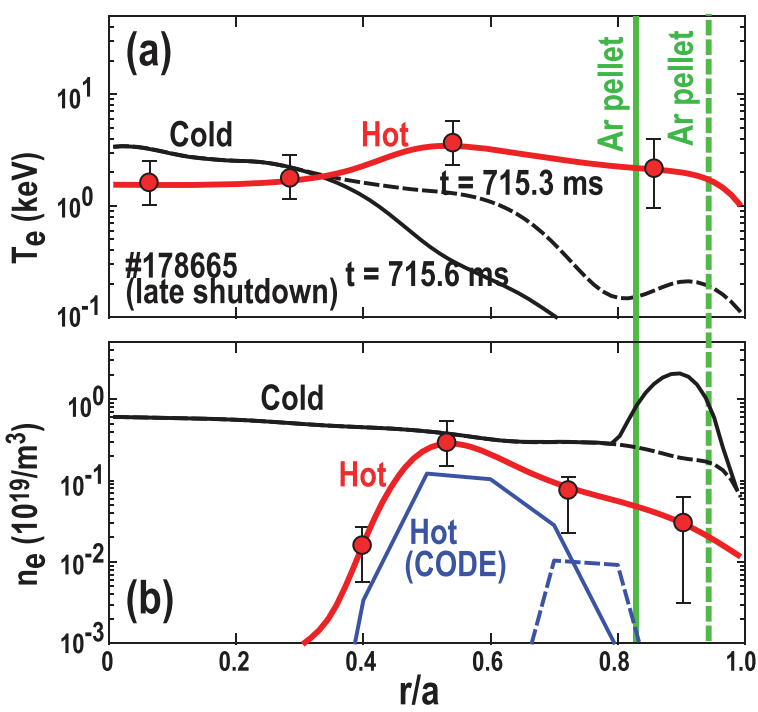

FIG. 10. Reconstructed radial profiles of (a) thermal and non-thermal electron temperature and $(b)$ thermal and non-thermal electron density at two times steps during the late shutdown shot (one at the pellet first impact and one during the pre-TQ). Blue curves show CODE simulations. 

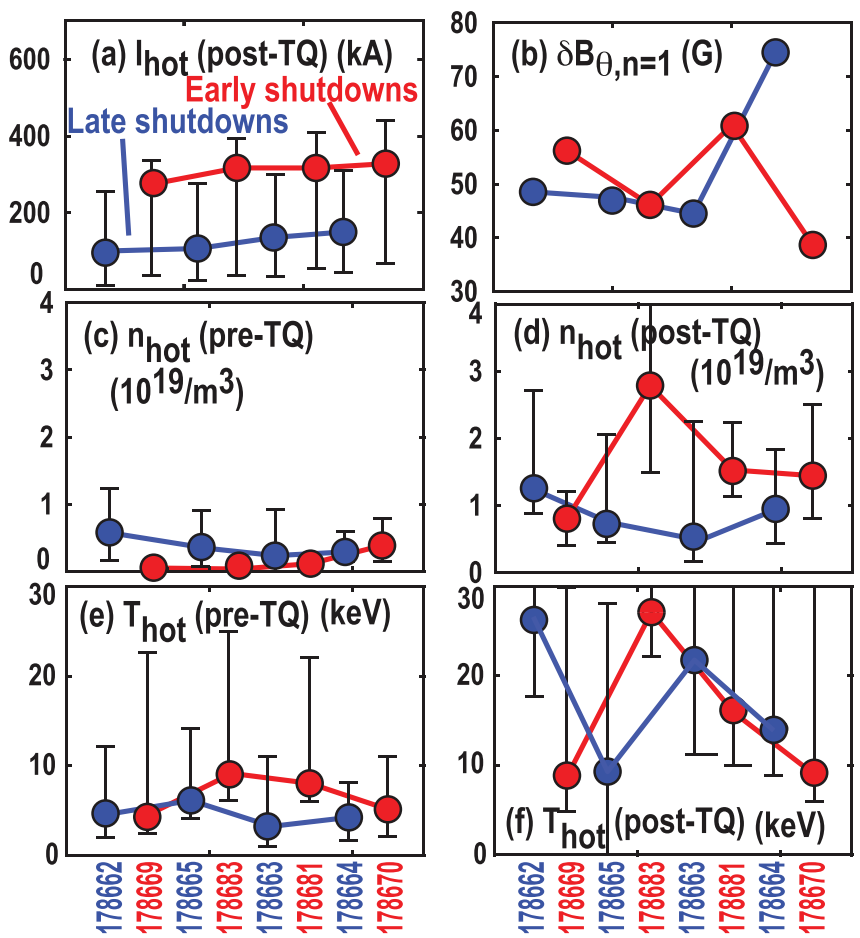

\section{Shot \#}

FIG. 11. Trends in similar shots showing four early shutdown and four late shutdown shots, ordered by increasing post-TQ RE current and showing: (a) post-TQ hot current estimated using avalanche method, (b) measured peak low-field side midplane wall $n=1$ poloidal magnetic fluctuation level, (c) profile-averaged hot density immediately before TQ onset estimated from ECE + SXR method, (d) profile-averaged hot density immediately after the $T Q$, (e) profile-averaged hot temperature immediately before $T Q$, and (f) profile-averaged hot temperature immediately after $T Q$.

slightly lower values for the early shutdowns. Figure 11(d) shows the profile-averaged hot electrons density immediately after the TQ, showing slightly higher values for the early shutdowns. Although hot density is not strictly equivalent to hot current, the apparent larger increase in hot electron density in the early shutdown cases is at least consistent with a trend of the early shutdowns having a larger increase in hot current during the TQ. Profile-averaged hot temperatures immediately before and after the TQ are shown in Figs. 11(e) and 11(f), showing typical temperatures of order $10 \mathrm{keV}$ before the TQ and of order $20 \mathrm{keV}$ after the TQ in both early and late shutdowns.

\section{EFFECT OF HOT ELECTRONS ON PELLET ABLATION}

The ECE + SXR reconstructed hot electron density profiles of Figs. 9 and 10 indicate that hot electrons are (a) either already present in the plasma when the argon pellet hits (for early shutdown) or (b) appear ahead of the pellet during the shutdown (for late shutdown). In either case, the pellet encounters plasma with hot electrons present for most of its trajectory through the plasma. This is supported by comparing the measured argon pellet ablation rate with the theoretical ablation rate of cryogenic argon pellets. Existing pellet ablation theory $^{32-34}$ does not treat plasmas with non-Maxwellian or two temperature electron distributions. Also, the theory assumes surface heat deposition, which begins to lose validity at $T_{e} \approx 10 \mathrm{keV}$, where the tail of the distribution, which is doing most of the ablation, has electron energies of $20 \mathrm{keV}$ and has non-negligible penetration depth. As electrons approach relativistic energies, they are expected to pass entirely through the pellet and heat it volumetrically. ${ }^{35}$ In previous work, CQ ablation of argon pellets was shown to be dominated by hot electrons and an approximate model of argon pellet ablation in the limit of fully penetrating relativistic hot electrons was used; ${ }^{11}$ which, however, is not expected to be valid here either. Despite the shortcomings of present pellet ablation modeling, the shape of the measured ablation curves is much closer to the predicted hot electron ablation curve than the predicted cold electron ablation curve, consistent with significant hot electrons existing at the argon pellet and dominating the ablation. This is shown in Fig. 12 by measured and predicted pellet ablation curves for (a) the early shutdown and (b) the late shutdown. For the theory curves, the reconstructed hot and cold electron ablation rates are put into the ablation model separately, i.e., no effort is made here to construct a self-consistent two temperature ablation model at this point.

\section{DISCUSSION}

The ECE + SXR analysis indicates that hot electrons appear ahead of the incoming Ar pellet. The formation of a thermal "cold
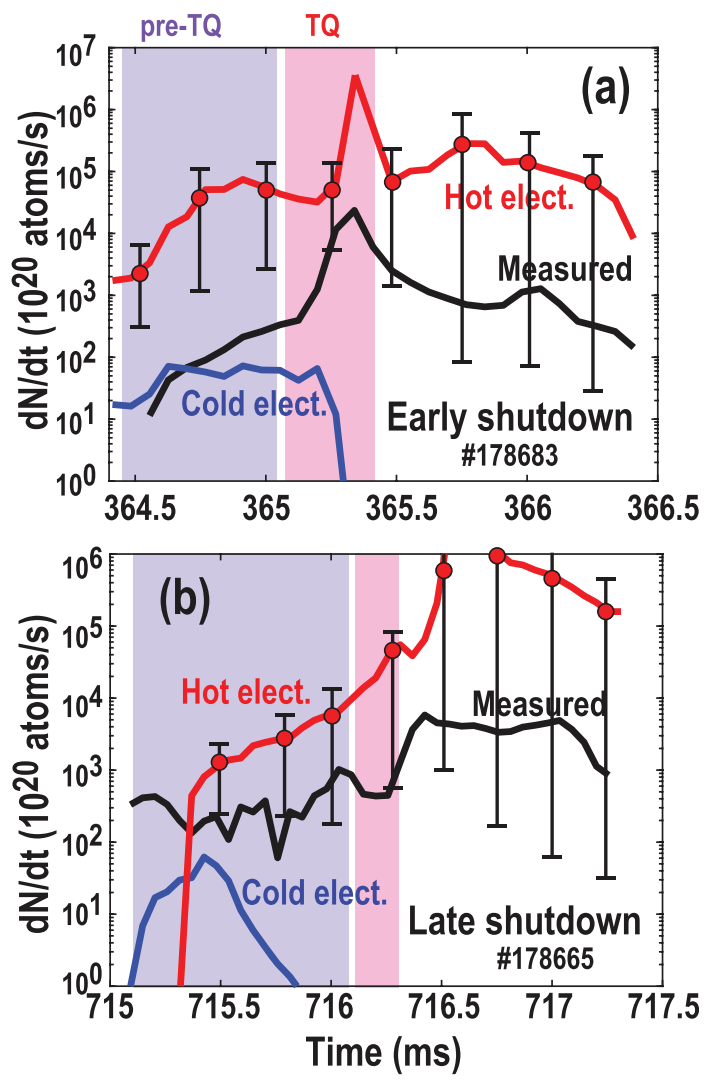

FIG. 12. Measured and predicted argon pellet ablation rates from hot and cold electrons vs time for (a) early shutdown and (b) late shutdown. 
front" moving ahead of Ar pellets has been shown previously; ${ }^{11}$ this work indicates that hot electrons exist in this cold front. The presence of hot electrons in the cold front could be either due to (a) Ar ions diffusing ahead of the Ar pellet, causing local thermal temperature collapse and local hot electrons formation or (b) rapid radial transport of hot electrons from behind the pellet inward past the pellet. Experimentally, these two possibilities cannot be separated with confidence with the present data. The simulations seem to favor option (a), since reasonable agreement between simulated pre-TQ hot electron density profiles and data is obtained with a local model (CODE), which does not include any fast radial transport of electrons. On the other hand, imposing a reasonable Ar ion radial diffusion coefficient $\left(5 \mathrm{~m}^{2} / \mathrm{s}\right)$ does not predict that Ar ions will move ahead of the pellet as far as the observed cold front, as demonstrated in Fig. 8(b), which tends to suggest rapid radial heat transport.

The CODE simulations of early and late shutdowns can also provide estimates of the hot current density profiles during the shutdowns. Figure 13 shows the measured and CODE modeled profileaveraged hot electron density vs time for the early shutdown, Fig. 13(a), and for the late shutdown, Fig. 13(c). Error bars on the measured hot electron density are assigned based on ECE + SXR fit
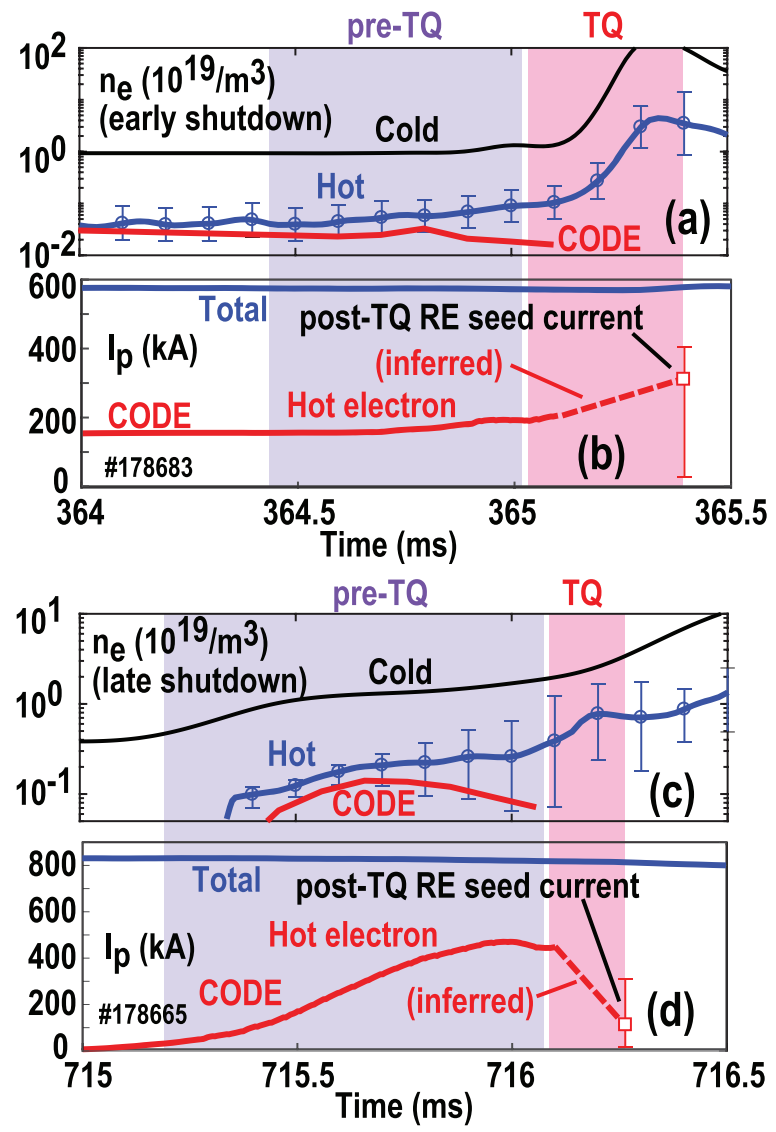

FIG. 13. Time traces of measured and predicted profile-average hot electron density (measured and simulated), as well as resulting hot electron current (simulated) for early shutdown case [(a) and (b)] and late shutdown case [(c) and (d)]. quality. It can be seen that the measured and simulated profileaveraged hot electron densities are in reasonable agreement (within $2 \times$ in the middle of the pre-TQ where the ECE + SXR method used here is expected to give best results), lending credence to the possibility that the CODE predicted hot electron current, Figs. 13(b) and 13(d), could have some validity. This predicted hot electron current is below the inferred (using RE plateau current and avalanche theory) post-TQ hot electron current in the early shutdown, Fig. 13(b), indicating a net gain in hot electron current during the TQ. Conversely, the predicted hot electron current ends above the inferred post-TQ hot electron current for the late shutdown, indicating a net loss of hot electron current during the TQ, Fig. 13(d). This contrasts with the typical picture in DIII-D, where the loss of hot electron current during the TQ is thought to be small enough $(\sim 20 \%)$ to be negligible, and the TQ is thought of as being a net source of hot electron current. ${ }^{11,12}$ It should be noted though that the error bars in the inferred post-TQ current are quite large; these are based in Fig. 13 on the large uncertainty in the CQ Dreicer gain and the smaller uncertainty in the CQ impurity density.

The ability of the TQ to cause net loss or gain of hot electrons is demonstrated, at least theoretically, in Fig. 14, which shows DREAM simulations of the late shutdown shot with varying levels of hot electron ("Rechester-Rosenbluth") TQ cross field diffusion ${ }^{36}$ added to the simulation. Three cases, $D_{R R}=0,10^{3}$, and $10^{4} \mathrm{~m}^{2} / \mathrm{s}$, are simulated. $D_{R R}=10^{3}$ and $10^{4} \mathrm{~m}^{2} / \mathrm{s}$ correspond to fully stochastic normalized perturbed magnetic field levels of order $\delta \tilde{B} \approx 10^{-3}$ and $3 \times 10^{-3}$. Normalized magnetic field perturbation levels of $\delta \tilde{B} \approx 10^{-3}$ are typically believed to exist in tokamaks at the end of the TQ MHD, ${ }^{37}$ so these values are not entirely unreasonable. DREAM is a FokkerPlanck solver (with non-linear solver and linear collision operator), which includes 1D (radial) hot electron diffusion and a self-consistent Ampère-Faraday solver for the electric field profile. ${ }^{38}$ Consistent with the CODE simulation of Fig. 13(d), DREAM predicts that the late shutdown forms a hot electron current of around $400 \mathrm{kA}$ during the

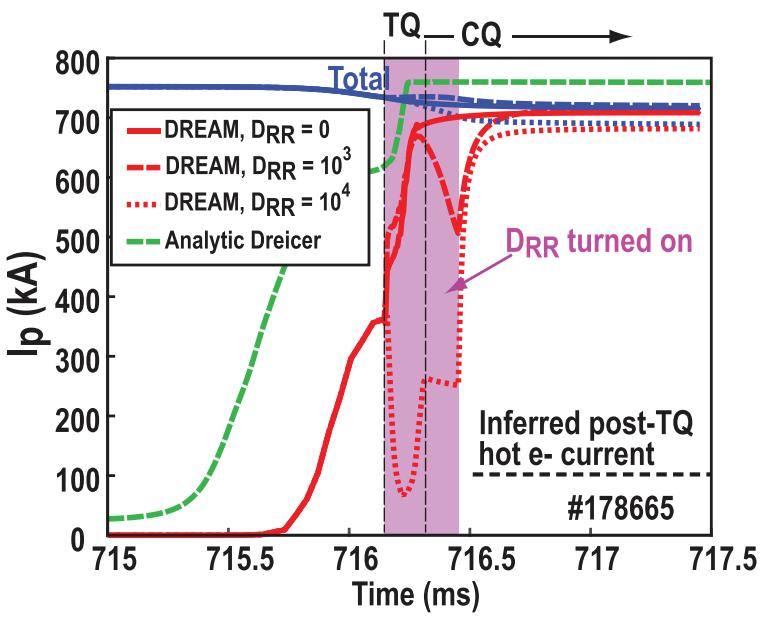

FIG. 14. Simulated total (blue) and hot (red) electron current vs time from 1D radially resolved Fokker-Planck DREAM simulation of late shutdown shot for three different levels of TQ hot electron radial transport. Radial diffusion is enabled in the shaded region and is characterized by diffusion coefficient $D_{R R}\left(\mathrm{~m}^{2} / \mathrm{s}\right)$. Green curve shows analytic Dreicer model for comparison. 
pre-TQ phase. With no radial loss, the TQ results in a gain of hot electron current, with a rise to about $700 \mathrm{kA}$ predicted $\left(D_{R R}=0\right.$ curve in Fig. 14). With a strong radial loss $\left(D_{R R}=10^{4} \mathrm{~m}^{2} / \mathrm{s}\right)$, a decrease in hot electron current to about $250 \mathrm{kA}$ is predicted in Fig. 14, thus demonstrating that the level of TQ hot electron loss can determine if the TQ results in net gain or loss of hot electron current. In these simulations, $D_{R R}$ is turned on at the TQ onset and turned off $0.1 \mathrm{~ms}$ after the TQ ends; this "turn off time" is consistent with NIMROD simulations of TQ MHD, giving a $\sim 0.1 \mathrm{~ms}$ timescale for stochastic field line healing post-TQ. ${ }^{8}$ The DREAM simulations indicate that Dreicer formation becomes significant during the CQ of this shot, resulting in nearly complete ( $>90 \%$ ) hot electron current conversion in all cases during the CQ. This was double-checked with a semianalytic Dreicer model, which uses the analytic Dreicer expression for Drecier seed formation, integrated over the plasma radial profile during the disruption. In both DREAM and analytic Dreicer models, the initial electric field during the shutdown is estimated from the initial measured current density profile; although the models differ in that DREAM includes subsequent radial diffusion of the current density and also the drag on the hot electrons, while the analytic model does not. The Dreicer model is shown by the green-dashed curve in Fig. 14 and, consistent with the DREAM simulations, gives nearly full RE conversion. This is clearly in contrast with the experiment, which infers a post-TQ hot electron current level of about $100 \mathrm{kA}$ and very little hot electron current gain during the CQ. The reason for this discrepancy is not understood yet. Dreicer hot electron formation is extremely sensitive to density, as well as CQ temperature (and resulting electric field), so it is possible that the prediction of full CQ Dreicer formation is a result of uncertainties in the radial distribution of argon in the plasma. A different possible explanation is that Dreicer hot electron formation is indeed occurring here during the $\mathrm{CQ}$, but there is a hot electron radial loss term that counteracts the Dreicer seed. Ignoring the CQ Dreicer issue for now, the DREAM simulations indicate that a huge diffusion coefficient $D_{R R} \approx 10^{4} \mathrm{~m}^{2} / \mathrm{s}$ is needed to give post-TQ hot electron currents of order $100 \mathrm{kA}$, as inferred from avalanche theory. This corresponds to a hot electron loss timescale $<0.1 \mathrm{~ms}$, so less than the TQ duration, and faster than the avalanche timescale (of about $2 \mathrm{~ms}$ ). This indicates that the small ( 20\%) TQ hot electron loss fractions previously estimated for DIII-D disruptions may underestimate TQ hot electron loss in these shots.

Quantifying the level of hot electron radial loss during disruptions experimentally is notoriously challenging. Peak magnetic fluctuation levels measured at the wall are shown in Fig. 11(b), but show no clear trends. In principle, the late shutdown cases, with higher $I_{P}$ and lower $q$, can be expected to have larger TQ MHD and larger associated hot electron loss, but this does not appear to be reflected by higher levels of poloidal magnetic field fluctuations measured at the wall. Todate, it is not well-established what the best experimental signal is to quantify TQ MHD loss of hot electrons, and it is likely that $n=1$ magnetic perturbations at the wall are not a good indicator of hot electron TQ loss. Other harmonics (higher than $n=1$ ) are not shown in Fig. 11(b); they also show no clear correlation with RE loss in these experiments. There does appear to be a higher hot electron density gain during the early shutdown cases, Fig. 11(d), consistent with decreased hot electron loss (from the higher $q$ ) and/or increased hot tail seed formation (from the higher $T_{e}$ ). Overall, however, it is not possible at present to experimentally confirm the presence of a huge TQ hot electron loss term in these shots.

\section{SUMMARY}

In summary, pre-TQ hot electron density profiles are reconstructed by combining ECE and SXR profile analysis for low-density circular target plasmas shut down by cryogenic Ar pellet injection. The technique introduced here, of separating cold and hot electron ECE emission using an exponential decay fit, appears to be reasonably valid for these shots, as indicated by cross-comparison with TS data. This technique is limited in applicability to the pre-TQ phase of lowdensity plasmas, as low density is needed to avoid ECE cutoff. During the TQ and CQ, Ar impurities can arrive in significant densities at the SXR diagnostic location, changing $Z_{\text {eff }}$ in an unpredictable fashion. Also, hot electron ECE emission becomes less localized, leading to eventual harmonic overlap, making the ECE emission increasingly hard to interpret.

The ECE + SXR analysis indicates that the plasma thermal temperature collapses and hot electrons appear ahead of the incoming Ar pellet during the pre-TQ. At present, it is not certain if this is due to Ar ions transporting ahead of the pellet or hot electrons transporting ahead of the pellet. Evidence for rapid radial impurity transport during massive gas injection shutdowns has been seen previously both experimentally ${ }^{27}$ and theoretically, ${ }^{39}$ so it is not unreasonable to expect that rapid radial impurity transport also occurs during pellet injection shutdowns. In any case, the hot electrons forming ahead of the pellet are shown to dominate the pellet ablation, thus strongly affecting the shutdown impurity deposition profile. This is a negative result for disruption mitigation by impurity injection, as it indicates that reaching the core of large tokamaks with injected pellets could be harder than expected.

Fokker-Planck modeling of these cases indicates that the hot electron current can already be quite significant (up to 50\% of the total current) in the pre-TQ phase, highlighting the importance of selfconsistent electric field modeling for hot electron formation prediction, even during the pre-TQ. Comparison between the Fokker-Planck modeling and the post-TQ hot electron current estimated from avalanche theory suggests that a net loss of hot electron current can occur during the TQ in some cases. This interesting finding, if true, indicates that TQ hot electron loss can be quite large even in mid-sized tokamaks like DIII-D and underscores the need for further modeling and diagnosis of disruption hot electron radial loss. However, this finding is extremely preliminary because of large uncertainties in both the pre-TQ hot electron current and post-TQ hot electron current, as described above. Future work will continue to try to improve measurements and modeling of the important effect of the TQ on hot electron current.

In terms of relevance to disruption mitigation in future large tokamaks (such as the ITER baseline scenario), some aspects of this work are expected to be relevant, while others are most likely not relevant. The initial temperatures (up to $10 \mathrm{keV}$ ) studied here are comparable to ITER, while the electron densities studied here are far lower, and plasma dimensions are significantly smaller. The finding that impurity pellet injection into hot current-carrying plasma creates hot electrons ahead of the pellet, which increase the pellet ablation rate, could very well be relevant for ITER disruption mitigation using neon pellets, as already suggested in recent neon pellet analysis in DIII-D. ${ }^{40}$ This would depend on the level of impurity ion and/or fast electron transport moving ahead of the strongly perturbing neon pellet and is unknown presently for ITER. The possible implication of this work 
that TQ hot electron loss in DIII-D may be more significant than expected may be relevant for ITER. Presently, TQ hot electron loss is expected to be small in ITER, but this is based entirely on modeling; so if simulations of DIII-D underestimate TQ hot electron loss in DIII-D, this very well also be the case in ITER. The finding here that Dreicer hot electron production could be significant in the CQ of these DIII-D shots is expected to be a peculiarity of the low densities used here; in ITER, the much larger CQ densities are expected to completely suppress the Dreicer seed. Similarly, the much higher density in ITER is expected to quickly remove any startup slide-away electrons, so the finding here that pre-existing hot electrons can reduce additional hot electron formation is probably only of interest to analysis of disruptions in very low-density tokamak discharges.

\section{ACKNOWLEDGMENTS}

The authors gratefully acknowledge the advice of T. Fülöp on CODE and DREAM simulations. Permission to use PrismSPECT from I. Golovkin is gratefully acknowledged. This material is based upon work supported by the U.S. Department of Energy, Office of Science, Office of Fusion Energy Sciences, using the DIII-D National Fusion Facility, a DOE Office of Science user facility, under Award Nos. DE-FG02-07ER54917, DE-FC02-04ER54698, DE-AC05-00OR22725, DE-AC52-07NA27344, DEFG02-04ER54744, and DE-AC05-06OR23100, as well as on work supported by the Swedish Research Council under Award No. 2018-03911. This report was prepared as an account of work sponsored by an agency of the United States Government. Neither the United States Government nor any agency thereof, nor any of their employees, makes any warranty, express or implied, or assumes any legal liability or responsibility for the accuracy, completeness, or usefulness of any information, apparatus, product, or process disclosed, or represents that its use would not infringe privately owned rights. Reference herein to any specific commercial product, process, or service by trade name, trademark, manufacturer, or otherwise does not necessarily constitute or imply its endorsement, recommendation, or favoring by the United States Government or any agency thereof. The views and opinions of authors expressed herein do not necessarily state or reflect those of the United States Government or any agency thereof.

\section{DATA AVAILABILITY}

The data that support the findings of this study are available from the corresponding author upon reasonable request. Additionally, DIII-D data shown in this paper can be obtained in digital format by following the links at Ref. 41 .

\section{REFERENCES}

${ }^{1}$ M. Lehnen, K. Aleynikova, P. B. Aleynikov, D. J. Campbell, P. Drewelow, N. W. Eidietis, Y. Gasparyan, R. S. Granetz, Y. Gribov, N. Hartmann, E. M. Hollmann, V. A. Izzo, S. Jachmich, S. H. Kim, M. Kocan, H. R. Koslowski, D. Kovalenko, U. Kreuzi, A. Loarte, S. Maruyama, G. F. Matthews, P. B. Parks, G. Pautasso, R. A. Pitts, C. Reux, V. Riccardo, R. Roccella, J. A. Snipes, A. J. Thornton, P. C. de Vries, and EFDA JET Contributors, J. Nucl. Mater. 463, 39 (2015).

${ }^{2}$ E. M. Hollmann, P. B. Aleynikov, T. Fülöp, D. A. Humphreys, V. A. Izzo, M. Lehnen, V. E. Lukash, G. Papp, G. Pautasso, F. Saint-Laurent, and J. A. Snipes, Phys. Plasmas 22, 021802 (2015).
${ }^{3}$ H. Knoepfel and D. A. Spong, Nucl. Fusion 19, 785 (1979).

${ }^{4}$ ITER Physics Expert Group on Disruptions, Plasma Control, and MHD, Nucl. Fusion 39, 2251 (1999); available at http://iopscience.iop.org/0029-5515/39/12/303.

${ }^{5}$ T. C. Hender, J. C. Wesley, J. Bialek, A. Bondeson, A. H. Boozer, R. J. Buttery, A. Garofalo, T. P. Goodman, R. S. Granetz, Y. Gribov et al., Nucl. Fusion 47, S128 (2007)

${ }^{6}$ H. M. Smith and E. Verwichte, Phys. Plasmas 15, 072502 (2008).

${ }^{7}$ B. N. Breizman, P. Aleynikov, E. M. Hollmann, and M. Lehnen, Nucl. Fusion 59, 083001 (2019).

${ }^{8}$ V. A. Izzo, D. A. Humphreys, and M. Kornbluth, Plasma Phys. Controlled Fusion 54, 095002 (2012).

${ }^{9}$ C. Sommariva, E. Nardon, P. Beyer, M. Hoelzl, G. T. A. Huijsmans, and JET Contributors, Nucl. Fusion 58, 106022 (2018).

${ }^{10}$ V. A. Izzo, E. M. Hollmann, A. N. James, J. H. Yu, D. A. Humpreys, L. L. Lao, P. B. Parks, P. E. Sieck, J. C. Wesley, R. S. Granetz, G. M. Olynyk, and D. G. Whyte, Nucl. Fusion 51, 063032 (2011).

${ }^{11}$ E. M. Hollmann, N. Commaux, R. A. Moyer, P. B. Parks, M. E. Austin, I. Bykov, C. Cooper, N. W. Eidietis, M. O'Mullane, C. Paz-Soldan, D. L. Rudakov, and D. Shiraki, Nucl. Fusion 57, 016008 (2017).

${ }^{12}$ C. Paz-Soldan, P. Aleynikov, E. M. Hollmann, A. Lvovskiy, I. Bykov, X. Du, N. W. Eidietis, and D. Shiraki, Nucl. Fusion 60, 056020 (2020).

${ }^{13}$ A. Lvovskiy, C. Paz-Soldan, N. W. Eidietis, A. Dal Molin, X. D. Du, L. Giacomelli, J. L. Herfindal, E. M. Hollmann, L. Martinelli, R. A. Moyer, M. Nocente, D. Rigamonti, D. Shiraki, M. Tardocchi, and K. E. Thome, Plasma Phys. Controlled Fusion 60, 124003 (2018)

${ }^{14}$ M. N. Rosenbluth and S. V. Putvinski, Nucl. Fusion 37, 1355 (1997).

${ }^{15}$ H. Dreicer, Phys. Rev. 115, 238 (1959).

${ }^{16}$ A. N. James, M. E. Austin, N. Commaux, N. W. Eidietis, T. E. Evans, E. M. Hollmann, D. A. Humphreys, A. W. Hyatt, V. A. Izzo, T. C. Jernigan, R. J. La Haye, P. B. Parks, E. J. Strait, G. R. Tynan, J. C. Wesley, and J. H. Yu, Nucl. Fusion 52, 013007 (2012).

17. L. Luxon, Nucl. Fusion 42, 614 (2002).

${ }^{18}$ M. E. Austin and J. Lohr, Rev. Sci. Instrum. 74, 1457 (2003).

${ }^{19}$ E. M. Hollmann, L. Chousal, R. K. Fisher, R. Hernandez, G. L. Jackson, M. J. Lanctot, S. V. Pidcoe, J. Shankara, and D. A. Taussig, Rev. Sci. Instrum. 82, 113507 (2011).

${ }^{20}$ L. Hesslow, O. Embréus, O. Vallhagen, and T. Fülöp, Nucl. Fusion 59, 084004 (2019).

${ }^{21}$ N. Bosviel, P. Parks, and R. Samulyak, Phys. Plasmas 28, 012506 (2021).

${ }^{22}$ F. L. Hinton and C. Oberman, Nucl. Fusion 9, 319 (1969).

${ }^{23}$ O. Sauter, C. Angioni, and Y. R. Liu-Liu, Phys. Plasmas 6, 2834 (1999).

${ }^{24} \mathrm{~J}$. Wesson and D. J. Campbell, Tokamaks (Oxford University Press, Oxford, 2011).

${ }^{25}$ M. Landreman, A. Stahl, and T. Fülöp, Comput. Phys. Commun. 185, 847 (2014).

${ }^{26}$ A. Stahl, O. Embreus, G. Papp, M. Landreman, and T. Fülöp, Nucl. Fusion 56, 112009 (2016).

${ }^{27}$ E. M. Hollmann, T. C. Jernigan, P. B. Parks, J. A. Boedo, T. E. Evans, M. Groth, D. A. Humphreys, A. N. James, M. J. Lanctot, D. Nishijima, D. L. Rudakov, H. A. Scott, E. J. Strait, M. A. Van Zeeland, J. C. Wesley, W. P. West, W. Wu, and J. H. Yu, Nucl. Fusion 48, 115007 (2008).

${ }^{28}$ M. Bornatici, R. Cano, O. De Barbieri, and F. Engelmann, Nucl. Fusion 23, 1153 (1983).

${ }^{29}$ M. E. Austin, R. F. Ellis, and T. C. Luce, "Determination of wall reflectivity for ECE frequencies in DIII-D," in Proceedings of the 10th ECE/ECRH Workshop, Ameland, Netherlands (World Scientific, 1997), p. 249.

${ }^{30}$ W. Halverson, Plasma Phys. 14, 601 (1972).

${ }^{31}$ J. E. Bailey, G. A. Rochau, R. C. Mancini, C. A. Iglesias, J. J. MacFarlane, I. E. Golovkin, C. Blancard, P. Cosse, and G. Faussurier, Phys. Plasmas 16, 058101 (2009).

${ }^{32}$ P. Parks, N. Bosviel, and R. Samulyak, "The ablation rate of light-element pellets with a kinetic treatment for penetration of plasma electrons through the ablation cloud," Phys. Plasmas (unpublished) (2021).

${ }^{33}$ P. B. Parks and M. N. Rosenbluth, Phys. Plasmas 5, 1380 (1998).

${ }^{34}$ V. Y. Sergeev, Plasma Phys. Rep. 32, 363 (2006).

${ }^{35}$ D. I. Kiramov and B. N. Breizman, Nucl. Fusion 60, 084004 (2020).

${ }^{36}$ A. B. Rechester and M. N. Rosenbluth, Phys. Rev. Lett. 40, 38 (1978). 
${ }^{37}$ J. A. Wesson, R. D. Gill, M. Hugon, F. C. Schuller, J. A. Snipes, D. J. Ward, D. V. Bartlett, D. J. Camplbell, P. A. Duperrex, A. W. Edwards, R. S. Granetz, N. A. O. Gottardi, T. C. Hender, E. Lazzaro, P. J. Lomas, N. Lopes Cardozo, K. F. Mast, M. F. F. Nave, N. A. Salomon, P. Smeulders, P. R. Thomas, B. J. D. Tubbing, M. F. Turner, and A. Weller, Nucl. Fusion 29, 641 (1989).

${ }^{38} \mathrm{O}$. Embreus, M. Hoppe, and T. Fülöp, "DREAM: A fluid-kinetic framework for tokamak disruption runaway electron simulations,” arXiv:2103.16457 (2021).
${ }^{39}$ V. A. Izzo, D. G. Whyte, R. S. Granetz, P. B. Parks, E. M. Hollmann, L. L. Lao, and J. C. Wesley, Phys. Plasmas 15, 056109 (2008).

${ }^{40}$ E. M. Hollmann, D. Shiraki, L. Baylor, I. Bykov, N. W. Eidietis, I. Golovkin, J. L. Herfindal, A. Lvovskiy, A. McLean, R. A. Moyer, T. O'Gorman, P. B. Parks, and Z. Popovic, Nucl. Fusion 61, 016023 (2021).

${ }^{41}$ See https://fusion.gat.com/global/D3D_DMP for DIII-D Data Management Plan. 